\title{
Impacto do diagnóstico pré-natal (DPN) citogenético e ansiedade materna sobre a interação precoce mãe-bebê
}

\author{
The impact of cytogenic prenatal diagnosis (PND) and maternal anxiety on early mother-child interaction
}

Impacto del diagnóstico prenatal (DPN) citogenético y ansiedad maternal sobre la interacción precoz madre-bebé

Maria do Céu Diogo Nunes ${ }^{1}$

\footnotetext{
${ }^{1}$ Enfermeira, Doutora em Ciências de Enfermagem. Enfermeira Chefe da Maternidade Bissaya Barreto Maternidade Bissaya Barreto (Centro Hospitalar e Universitário de Coimbra, EPE).Coimbra, Portugal. E-mail: ceudnunes@gmail.com.
}

\section{RESUMO}

O presente estudo de natureza longitudinal teve como objetivo estudar a influência que o diagnóstico pré-natal citogenético pode exercer sobre a ansiedade materna, a adaptação à gravidez e a relação precoce mãe-bebê, particularmente em grávidas de idade materna avançada. A amostra incidiu sobre 3 grupos de gestantes: G1 selecionadas para amniocentese ( $\geq 35$ anos); G2 selecionadas para amniocentese por rastreio (<35 anos); G3 gestantes (20-34 anos) não selecionadas, e decorreu entre Março de 2008 e Maio de 2009. Os resultados revelaram que a expectativa de realização do exame citogenético conduz a um aumento da ansiedade-estado mais significativo entre as mulheres propostas para teste após rastreio positivo; a ansiedade-estado evidenciada pelas mães é mediadora da relação entre a interação mãe-bebê e os sentimentos face à gravidez. Estes resultados permitem fundamentar recomendações no sentido de efetuar uma intervenção profilática entre as mães que, durante a gravidez, reagiram com maior ansiedade ao exame.

Descritores: Gravidez; Amniocentese; Ansiedade; Recém-nascido; Comportamento Materno.

\section{ABSTRACT}

The objective of this longitudinal study was to study the influence of cytogenic prenatal diagnosis on maternal anxiety, her adaptation to pregnancy and on early mother-child relation, particularly in pregnant women at a mature age. The sample was formed by three groups of pregnant women: G1selected for amniocentesis ( $\geq 35$ years old); G2 selected for amniocentesis through screening (<35 years old); G3 pregnant women not selected (20-34 years old). The study was performed between March of 2008 and May of 2009. The results revealed that the expectation to take the cytogenic test increases anxiety-state more significantly among women referred to tests after a positive screening; the anxiety-state evidenced by mothers is a mediator of the mother-child interaction and feelings towards the pregnancy. These results permit to found recommendations in the sense of making a prophylactic intervention among mothers who, during pregnancy, reacted to the test with greater anxiety.

Descriptors: Pregnancy; Amniocentesis; Anxiety; Infant, Newborn; Maternal Behavior.

\section{RESUMEN}

Este estudio longitudinal objetivó identificar la influencia del diagnóstico prenatal citogenético en la ansiedad maternal, la adaptación al embarazo y la relación precoz madre-bebé, particularmente en embarazadas de edad materna avanzada. La muestra incidió sobre tres grupos de gestantes: G1 seleccionadas para amniocentesis criterios etarios ( $\geq 35$ años); G2 seleccionadas para amniocentesis por rastreo (<35 años); G3 gestantes (20-34 años) no seleccionadas, y transcurrió entre marzo 2008 y mayo 2009. Los resultados expresaron que la expectativa de realización del examen citogenético lleva a un aumento de la ansiedad-estado, más significativo entre las mujeres propuestas para el test luego de rastreo positivo; la ansiedad-estado expresada por las madres es mediadora de la relación entre la interacción madre-bebé y los sentimientos frente al embarazo. Estos resultados permiten fundamental recomendaciones en el sentido de efectuar una intervención profiláctica entre las madres que, durante el embarazo, reaccionaron con mayor ansiedad al examen.

Descriptores: Embarazo; Amniocentesis; Ansiedad; Recién Nacido; Conducta Materna. 


\section{INTRODUÇÃO}

O aumento do número de gestações acima dos 35 anos de idade é uma realidade cada vez mais importante, sobretudo nos países mais desenvolvidos. Com efeito, cada vez mais mulheres adiam a decisão de ter filhos, especialmente nos países industrializados ${ }^{(1)}$.

Nos Estados Unidos estima-se que uma em cada cinco mulheres tem o seu primeiro filho após os 35 anos de idade. Em Portugal a taxa de gravidez em mulheres com idade materna avançada, passou de $5 \%$ nos anos setenta do século passado para $16,3 \%$ nos nossos dias(2).

No que se refere à idade média ao tempo do primeiro parto também se verificou um aumento: nos Estados Unidos para 25,2 anos, na Suécia para 28,3 anos e no Canadá para os 29,6 anos ${ }^{(3)}$.

Sem dúvida que estamos perante uma modificação profunda dos estilos de vida. Com efeito, se, no passado era comum encontrar mulheres de 20 anos já casadas e com filhos, hoje é habitual que só pelos 30 anos a mulher inicie o caminho que a leva à constituição de família. Outras mulheres esperam ainda um pouco mais, não sendo raro encontrar mulheres que só decidem ter filhos depois dos 40 anos de idade.

Entre as razões para tal tendência, os autores enunciam a ampla disponibilidade de métodos contraceptivos, a postergação pelo matrimônio, a maior incidência de divórcios, o desejo de atingir um nível educacional e profissional mais elevado, a necessidade de conquistar estabilidade e independência financeira, o aperfeiçoamento e generalização de técnicas de fertilização artificial, ou simplesmente, a dúvida sobre "ser ou não ser mãe"(1).

Sabe-se que a gravidez tardia traz consigo alguns riscos, tanto para a saúde da mãe como do feto. Assim, a idade materna avançada é hoje uma preocupação obstétrica quanto aos resultados maternos e perinatais, tendo em conta que a partir dos 35 anos de idade aumentam significativamente os riscos associados à gravidez, nomeadamente a ocorrência de cromossopatias, tais como a Síndrome de Down ${ }^{(3-4)}$. Esta afecção é provavelmente a doença mais frequente para a qual é solicitado o diagnóstico pré-natal (DPN) citogenético, mais concretamente a amniocentese.

A amniocentese é um método de diagnóstico invasivo que consiste na obtenção e análise de uma amostra de líquido amniótico (LA) através de uma punção na parede abdominal da mãe, sob controle ecográfico e prévia administração de anestesia local. O material utilizado para análise são as células fetais flutuantes no líquido amniótico, e algumas análises podem ser realizadas no sobrenadante ${ }^{(5)}$.

Embora a amniocentese seja uma técnica relativamente segura, de rápida execução e com uma acuidade diagnóstica muito elevada, existem algumas complicações a ela associadas, tanto para a mãe como para o feto, nomeadamente o traumatismo direto provocado pela agulha (feto, placenta, cordão umbilical e órgãos maternos), a infeção e a indução precoce do parto ou o abortamento(5).

Assim, a realização da amniocentese é uma experiência com profundo impacto psicológico, tendo em conta que deste procedimento diagnóstico pode derivar um diagnóstico fetal ou o risco de aborto(6). Deste modo, a tecnologia pré-natal, pelo seu caráter invasivo, interfere na qualidade da relação materno-fetal(7) e tende a acentuar a ansiedade da gestante relativamente ao bemestar do seu bebê(8-10). Tendo em conta o papel adverso que a sintomatologia ansiosa pode ter durante a gravidez, justifica-se a importância deste estudo sobre o impacto que os procedimentos do DPN, e em particular a amniocentese, podem exercer sobre a adaptação à gravidez e a relação mãe-bebê.

Os enfermeiros de saúde materna mantém uma relação privilegiada com a grávida ao longo de todo o ciclo gravídico, pelo que se torna particularmente importante reconhecerem as causas desencadeantes de processos de ansiedade nestas mulheres, para que possam contribuir para a sua diminuição, através do planejamento de intervenções adequadas a cada situação(11).

Este estudo desenvolve-se, assim, em torno do seguinte objetivo: avaliar o impacto que os procedimentos do DPN, e em particular a amniocentese, exercem sobre a ansiedade materna, os sentimentos face à gravidez e a qualidade da interação precoce mãe-bebê, particularmente em grávidas de idade materna avançada.

\section{MATERIAL E MÉTODOS}

O estudo de natureza longitudinal, foi concretizado em duas fases. O objetivo da fase I foi avaliar o impacto do DPN citogenético sobre a ansiedade materna e a adaptação à gravidez/maternidade; a fase II teve por 
objetivo investigar a influência da ansiedade sobre a relação precoce mãe-bebê.

O trabalho de campo repartiu-se por três momentos de avaliação (antes da amniocentese - T1 - depois da amniocentese - T2 - e no $2^{\circ}$ dia do pós-parto - T3).

A amostra foi constituída por três grupos escrutinados a partir de uma população constituída por mulheres grávidas ( $N=110)$, com idade gestacional entre as 12-18 semanas, sem filhos com doença genética a frequentarem as consultas de obstetrícia de uma maternidade pública em Coimbra (Portugal), e com condições de literacia que thes permitiam preencher os instrumentos do protocolo de investigação: G1 selecionadas para amniocentese por critérios etários (35 ou mais anos à data prevista para o parto); G2 selecionadas para amniocentese por rastreio, e com idade inferior a 35 anos à data prevista para o parto; G3 gestantes entre os 20 e 34 anos não selecionadas para amniocentese, no período de março de 2008 a maio de 2009.

Na definição da amostra foi utilizado um duplo critério: idade e exposição a procedimentos de DPN.

Consideraram-se critérios de inclusão: idade gestacional (12-18 semanas) em $\mathrm{T} 1 ; 1^{\mathrm{a}}$ amniocentese; relação marital estável; ter completado, pelo menos, o $1^{\circ}$ ciclo do ensino básico; frequentar as consultas de vigilância da gravidez na instituição; gravidez de feto único.

Como critérios de exclusão: patologia associada à gravidez; fetos e/ou recém-nascidos com malformações congênitas em gestações anteriores; gravidez após fecundação in-vitro (FIV); situações de perturbação de saúde mental.

A coleta de dados foi efetuada através de quatro instrumentos:1) State-Trait Anxiety Inventory - (STAl). Trata-se de uma escala de autoavaliação constituída por duas subescalas: escala Y1 - que avalia a Ansiedade Estado (o modo como o indivíduo se sente no momento 20 itens) e escala $Y 2$ - que avalia a Ansiedade Traço (o modo como o indivíduo se sente habitualmente - 20 itens) $^{(12)}$; 2) Pregnancy Research Questionnaire (PRQ). O PRQ é um questionário de autorrelato constituído por 37 itens, agrupados em seis escalas diferentes: Medos em relação ao Self; Desejo de Gravidez; Dependência; Medos em relação ao Bebê; Sentimento Maternal e Irritabilidade ${ }^{(13)}$. 3) "Protocolo de Observação da
Interação Mãe Bebê 0 a 6 meses" (POIMB) ${ }^{(14)}$. O POIMB 06 contém 21 itens, sendo 12 referentes ao comportamento da mãe e oito ao comportamento da criança durante a interação, bem como um item referente ao comportamento da díade (sintonia da interação); 4) Questionário de caracterização: dados sociodemográficos e obstétricos relativos à mãe e ao bebê.

A aplicação dos instrumentos (nos dois primeiros momentos de avaliação) efetuou-se enquanto as grávidas aguardavam por consulta médica de vigilância da gravidez, ou pelo exame diagnóstico.

Após o parto e certificação de que não havia ocorrido nenhuma alteração clínica grave que pudesse comprometer o desenvolvimento da criança, seguiu-se o contato com as mães no sentido de efetuar a gravação em vídeo das situações diádicas mãe-bebê.

As díades pertencentes ao estudo foram filmadas em condições idênticas. Рara a cotação dos 57 vídeos de interação mãe-bebê foi formada uma equipe de três juízes (dois mestrandos em Psicologia Clínica e uma enfermeira com formação na área de Saúde Materna) treinados na análise dos dados derivados da aplicação do instrumento de avaliação às puérperas, em estudo. Foi observada uma boa fidedignidade inter pares na cotação da grelha de avaliação. Todas as imagens de interação mãe-bebê foram pontuadas pelos observadores treinados sem nenhum conhecimento do grupo no qual os sujeitos se inseriam.

O projeto de pesquisa foi submetido à apreciação e aprovado pela Direção Clínica e Comissão de Ética da respetiva instituição de saúde (CE 10/2007). Antes do início do estudo as participantes foram informadas dos objetivos, sendo assegurada a confidencialidade e anonimato relativamente às informações fornecidas, tendo cada participante assinado documento de consentimento informado.

Para análise de dados, utilizaram-se técnicas da estatística descritiva e inferencial, recorrendo-se a testes não paramétricos sempre que se encontravam assegurados os pressupostos para a sua utilização (Teste de Kruskal-Wallis, Regressão linear e Teste de Sobel). O nível de significância estatística adotado foi de 0.05 . No tratamento da informação, utilizou-se o programa Statistical Package for Social Science - SPSS, versão 17.0. 


\section{RESULTADOS}

Participaram do estudo 110 mulheres grávidas, com idade gestacional entre as $12-18$ semanas, repartidas por três grupos: 49 no G1 (indicação DPN por idade), 19 no G2 (indicação DPN por rastreio) e 42 no G3 (sem indicação para DPN). Entre o início e o final do estudo, verificou-se uma perda amostral de 51,3\%, proporcional para todos os grupos.

As idades das participantes oscilaram entre os 20 e os 44 anos. Sendo que, para o G1 a média de idades foi 37,4 anos; para o G2 de 30,6 anos e para o G3 de 28,4 anos.

Quanto às características sociodemográficas, verificou-se uma certa homogeneidade entre os três grupos, sendo a maioria das mulheres casada, possuidora de nível escolar médio (considerando que a maioria completou o $10^{\circ} / 12^{\circ}$ ano de escolaridade), com ocupação profissional e de religião católica.

Na história obstétrica, e no que respeita à paridade, avaliada pelo número de filhos anteriores, verificou-se que a maioria das mulheres, de cada um dos grupos, já tinha, pelo menos um filho.

Para a maioria das mulheres o parto aconteceu por via vaginal: parto normal (eutócico) em 59,3\% dos casos no G1, 40,0\% no G2 e 45,0\% no G3; parto distócico instrumental, com recurso a fórceps (30,0\% no G2; $15,0 \%$ no G3 e 3,7\% no G1), ou a ventosa (20,0\% no G2; $15,0 \%$ no G3 e 11,1\% no G1), sendo que a maioria destas mulheres recorreu a analgesia epidural (80,0\% no G2; 80,0\% no G3 e $66,7 \%$ no G1).
A experiência do parto foi vivenciada conjuntamente com uma pessoa significativa para a maioria destas mulheres: $80,0 \%$ no G3; $77,8 \%$ no G2 e 70,4\% no G1, sendo o pai do bebê, a pessoa que mais vezes esteve presente.

A idade gestacional média na altura do parto foi de 39,1 semanas para o G3 (sem indicação DPN), 38,7 semanas para o G1 (indicação DPN por idade) e 38,6 para o G2 (indicação DPN por rastreio), não se verificando diferenças significativas entre os grupos $(p=0,468)$.

Em relação aos recém-nascidos, no que se refere ao sexo, verificou-se um certo equilíbrio, embora predominasse o sexo feminino. Na sua maioria bebês de termo, com bons índices de Apgar (8-10) e peso normal à nascença (entre $2500 \mathrm{gr}$ e $4000 \mathrm{gr}$ ).

Relativamente ao impacto do DPN citogenético sobre a ansiedade materna e a adaptação à gravidez/maternidade, no que se refere à ansiedade, verificaram-se diferenças estatisticamente significativas (Tabela 1) quanto à ansiedade-estado entre os três grupos ( $p=0,002$ ). As mulheres selecionadas por rastreio (G2) apresentaram maiores índices de ansiedade-estado antes da realização do exame (T1). Relativamente à ansiedade-traço não se verificaram diferenças estatisticamente significativas entre os três grupos de mulheres grávidas, antes da realização do DPN citogenético. No $2^{\circ}$ e no $3^{\circ}$ momento de avaliação (T2 e T3), após os resultados dos procedimentos de DPN e no pós-parto, os resultados obtidos apontaram para um decréscimo nos níveis de ansiedade.

Tabela 1: Comparação dos grupos em relação à ansiedade (traço e estado) antes e após os procedimentos de DPN (T1 e T2) e no pós parto (T3). Coimbra, Portugal, março de 2008 a maio de 2009.

\begin{tabular}{|c|c|c|c|c|c|c|c|}
\hline \multirow{3}{*}{ STAI } & \multirow{3}{*}{ Indicação DPN } & \multicolumn{6}{|c|}{ Momentos } \\
\hline & & \multicolumn{2}{|c|}{ T1 } & \multicolumn{2}{|c|}{ T2 } & \multicolumn{2}{|c|}{ T3 } \\
\hline & & Média & $\mathbf{p}^{*}$ & Média & p* & Média & $\mathbf{p}^{*}$ \\
\hline & (G1) por idade & 52,43 & & 42,56 & & 29,39 & \\
\hline \multirow[t]{3}{*}{ STAI-T } & (G2) por rastreio & 60,13 & 0,622 & 43,54 & 0,988 & 30 & 0,938 \\
\hline & (G3) Sem indicação & 56,99 & & 43,3 & & 27,98 & \\
\hline & (G1) por idade & 58,13 & & 39,91 & & 31 & \\
\hline \multirow[t]{2}{*}{ STAI-E } & (G2) por rastreio & 74,63 & 0,002 & 46,92 & 0,554 & 29,8 & 0,572 \\
\hline & (G3) Sem indicação & 43,77 & & 45,11 & & 25,9 & \\
\hline
\end{tabular}

*Teste Kruskall Wallis; $p<0.05$

No que diz respeito aos sentimentos face à gravidez verificaram-se diferenças estatisticamente significativas entre as grávidas do G2 (indicação de DPN por rastreio) e as do G1 (indicação de DPN por idade), nomeadamente ao nível dos "medos em relação ao Self" (receios em relação a si próprias) e da "dependência". As grávidas com indicação por rastreio (G2) apresentaram uma média mais elevada para a subescala "medos em relação ao Self" por comparação com as grávidas dos restantes grupos. Por outro lado, as grávidas com indicação por idade (G1) demonstraram níveis mais elevados de dependência (maior necessidade de apoio). Em relação às outras 
dimensões, não foram encontradas diferenças significativas. De fato, as grávidas com indicação de DPN (por idade ou rastreio) não diferiram relativamente aos seus receios em relação ao bebê, nem ao de desejo da gravidez, sentimento maternal ou irritabilidade. No terceiro trimestre da gravidez (T2), isto é, após o conhecimento, por parte das mulheres, dos resultados da amniocentese, não se verificaram diferenças significativas entre os grupos relativamente aos sentimentos face à gravidez (Tabela 2).

Tabela 2: Comparação dos grupos em relação aos sentimentos face à gravidez (PRQ) antes e após os procedimentos de DPN (T1 e T2). Coimbra, Portugal, março de 2008 a maio de 2009.

\begin{tabular}{|c|c|c|c|c|c|}
\hline \multirow{3}{*}{ PRQ } & \multirow{3}{*}{ Indicação DPN } & \multicolumn{4}{|c|}{ Momentos } \\
\hline & & \multicolumn{2}{|c|}{ T1 } & \multicolumn{2}{|c|}{ T2 } \\
\hline & & Média & $\mathbf{p}^{*}$ & Média & $\mathbf{p}^{*}$ \\
\hline & (G1) por idade & 52,94 & & 47,53 & \\
\hline \multirow[t]{3}{*}{ Medos em Relação ao Self } & (G2) por rastreio & 76,18 & 0,005 & 45,50 & 0,142 \\
\hline & (G3) Sem indicação & 49,13 & & 36,67 & \\
\hline & (G1) por idade & 61,12 & & 43,55 & \\
\hline \multirow[t]{3}{*}{ Desejo Gravidez ${ }^{1}$} & (G2) por rastreio & 49,47 & 0,233 & 36,19 & 0,532 \\
\hline & (G3) Sem indicação & 51,67 & & 45,03 & \\
\hline & (G1) por idade & 63,72 & & 47,23 & \\
\hline \multirow[t]{3}{*}{ Dependência } & (G2) por rastreio & 55,00 & 0,029 & 39,81 & 0,331 \\
\hline & (G3) Sem indicação & 46,13 & & 39,26 & \\
\hline & (G1) por idade & 61,40 & & 46,82 & \\
\hline \multirow[t]{3}{*}{ Medos Bebê } & (G2) por rastreio & 49,21 & 0,210 & 38,08 & 0,400 \\
\hline & (G3) Sem indicação & 51,46 & & 40,42 & \\
\hline & (G1) por idade & 57,28 & & 46,27 & \\
\hline \multirow[t]{3}{*}{ Sentimento Maternal² } & (G2) por rastreio & 52,18 & 0,824 & 38,15 & 0,488 \\
\hline & (G3) Sem indicação & 54,93 & & 41,05 & \\
\hline & (G1) por idade & 54,41 & & 44,44 & \\
\hline \multirow[t]{2}{*}{ Irritabilidade } & (G2) por rastreio & 59,39 & 0,837 & 41,31 & 0,881 \\
\hline & (G3) Sem indicação & 55,01 & & 41,97 & \\
\hline
\end{tabular}

*Teste Kruskall Wallis ; $p<0.05$

1. Quanto mais elevado o desejo da gravidez, menor a pontuação obtida.

2. Quanto mais elevado o sentimento maternal, menor a pontuação obtida.

Quanto à interação mãe-bebê não se verificaram diferenças significativas entre os grupos.

Para testar se a ansiedade exerce um efeito mediador sobre a relação entre os sentimentos face à gravidez (PRQ) e a interação mãe-bebê (POIMB), particularmente nas mulheres com indicação para DPN, o teste formal à existência de mediação consistiu na comparação de dois modelos de regressão linear, com posterior aplicação do teste de Sobel. Observou-se (Tabela 3) que a ansiedadeestado evidenciada pelas mães em T1 (antes da realização dos procedimentos de DPN/amniocentese) é mediadora da relação entre os sentimentos face à gravidez [(medos em relação ao bebê $(p=0,045)$ e irritabilidade $(p=0,040)$ ] e a interação mãe-bebê (competência materna envolvimento). A ansiedade-estado revelada pelas mães no segundo momento de avaliação (terceiro trimestre após resultados) possui uma associação significativa na relação entre a irritabilidade (PRQ) em T2 e a sensitividade (POIMB) ( $p=0,033)$; da relação entre os medos em relação ao bebê (PRQ), em T1 e T2 e o envolvimento (POIMB) $(p=0,040$ e $p=0,042$ respetivamente); e da irritabilidade em T2 e a competência materna envolvimento $(p=0,012)$. A ansiedade-estado em T2 é também mediadora da relação entre o sentimento maternal (PRQ) em T2 e a interação da díade (POIMB) $(p=0,012)$. Relativamente à ansiedadetraço evidenciada pelas mães em T3 ( $2^{\circ}$ dia do pós-parto), verificou-se que assume um papel mediador significativo na relação entre a irritabilidade (PRQ) em T1 $(p=0,049)$ e a sensitividade (POIMB) e na relação entre a dependência (PRQ) $(p=0,028)$ e a irritabilidade $(p=0,043)$, ambas em $\mathrm{T} 1$, e o envolvimento (POIMB). 
Tabela 3: Situações em que a ansiedade apresentou um papel mediador significativo entre sentimentos face à gravidez (PRQ) e interação mãe-bebê (POIMB), nas mulheres com indicação de DPN. Coimbra, Portugal, março de 2008 a maio de 2009.

\begin{tabular}{cccc}
\hline V. dependente & V. independente & V. mediadora & $\mathbf{p}^{*}$ \\
\hline POIMB_Envolvimento & PRQ_Medos Bebé (1) & STAI_Estado (1) & $\mathbf{0 , 0 4 5}$ \\
POIMB_Envolvimento & PRQ_Irritabilidade (1) & STAI_Estado (1) & $\mathbf{0 , 0 4 0}$ \\
POIMB_Sensitividade & PRQ_Irritabilidade (2) & STAI_Estado (2) & $\mathbf{0 , 0 3 3}$ \\
POIMB_Envolvimento & PRQ_Medos Bebê (1) & STAI_Estado (2) & $\mathbf{0 , 0 4 0}$ \\
POIMB_Envolvimento & PRQ_Medos Bebê (2) & STAI_Estado (2) & $\mathbf{0 , 0 4 2}$ \\
POIMB_Envolvimento & PRQ_Irritabilidade (2) & STAI_Estado (2) & $\mathbf{0 , 0 1 2}$ \\
POIMB_Díade & PRQ_Sentimento Maternal (2) & STAI_Estado (2) & $\mathbf{0 , 0 1 2}$ \\
POIMB_Sensitividade & PRQ_Irritabilidade (1) & STAI_Traço (3) & $\mathbf{0 , 0 4 9}$ \\
POIMB_Envolvimento & PRQ_Dependência (1) & STAI_Traço (3) & $\mathbf{0 , 0 2 8}$ \\
POIMB_Envolvimento & PRQ_Irritabilidade (1) & STAI_Traço (3) & $\mathbf{0 , 0 4 3}$ \\
\hline
\end{tabular}

* Teste Sobel

(1) - Antes dos procedimentos de DPN citogenético;

(2) - Depois dos procedimentos de DPN citogenético;

(3) $-2^{\circ}$ dia do pós-parto

\section{DISCUSSÃO}

No presente estudo partimos da hipótese que a execução de procedimentos de DPN citogenético, particularmente a amniocentese, tivessem influência na qualidade da interação precoce mãe-bebê (influência essa analisável através do impacto da ansiedade materna e dos sentimentos face à gravidez sobre os comportamentos interativos da mãe), sobretudo em mulheres com idade materna avançada. Todavia, o estudo efetuado revelounos que a execução de procedimentos de DPN citogenético parece não influenciar a interação mãebebê, já que não se verificaram diferenças significativas entre os grupos de mulheres grávidas. Estes resultados devem, no entanto, ser analisados cautelosamente e alguns dos pontos levantados no decurso do estudo devem ser considerados. De qualquer modo, as diferentes abordagens centradas nas consequências do DPN citogenético não diferem, de um modo geral, entre si. Em todas se admite que a gravidez vivenciada no contexto do DPN confronta os pais com desafios únicos ${ }^{(7-}$ 8,15-16). São escassos, porém, os estudos que abordam a relação precoce entre a mãe e o bebê no contexto do DPN citogenético, apontando-se assim a necessidade de novos estudos que corroborem ou não os achados do presente estudo.

Na verdade, face aos resultados poderemos supor que os procedimentos de diagnóstico pré-natal citogenético favorecem o estado ansioso das gestantes, essencialmente associado à dúvida que se instala da saúde e normalidade do bebê, sendo que esta consequência, no entanto, parece ser mais expressiva entre as mulheres propostas para amniocentese após um rastreio positivo. Os resultados favoráveis podem atenuar estes estados ansiosos ${ }^{(8-9,15)}$ - o que se veio a verificar no presente estudo, já que não foi detectada nenhuma anomalia ou malformação fetal promovendo a intensificação do vínculo.

Assim, a tranquilidade relativa proporcionada pelo conhecimento do resultado favorável do DPN citogenético contribui para um melhor ajustamento destas mulheres ao nascimento, como é referido na literatura ${ }^{(7-8,17)}$. Neste contexto, o parto surge como a confirmação final de que o bebê é perfeito, coincidindo portanto com o bebê imaginário constituído no início da gestação, isto é, em outras palavras, que o bebê real confirma os sonhos, e expectativas da mãe sobre o bebê sonhado.

Uma outra explicação possível para estes resultados pode ser dada à luz da perspetiva psicanalítica, pois a esperança sentida por parte da futura mãe de que o exame confirme que o feto é saudável, pode ser interpretado como mecanismo de defesa contra a ansiedade e a culpa por esta, sentida durante a gravidez. Este mecanismo de defesa protege a mulher grávida facilitando uma aproximação entre esta e o filho que aguarda o nascimento ${ }^{(18)}$.

Pelo seu lado, a ansiedade pré-natal e as fantasias maternas podem ser consideradas mecanismos saudáveis de ajustamento a um novo equilíbrio psicológico e que, neste contexto, as reações de alarme podem constituir uma espécie de tratamento de choque capaz de contribuir para a organização da mulher face ao seu novo

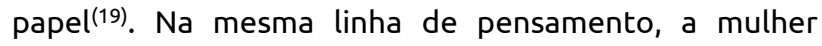
grávida ao sentir-se ameaçada pelo risco, reorganiza os 
seus mecanismos de defesa de forma a reprimir qualquer vivência impulsiva, anulando a vivência de possíveis conflitos psicológicos(20). Deste modo podemos presumir que as mulheres grávidas com indicação de DPN do nosso estudo, possam ter posto em ação mecanismos de defesa adequados à situação de risco vivenciada, e que, deste modo, tenham conseguido baixar gradualmente os níveis de ansiedade até ao pós-parto, com efeitos favoráveis no desenvolvimento das competências interativas com o seu bebê.

\section{CONCLUSÃO}

Ao abordar o fenômeno da gravidez e maternidade em idade materna avançada no contexto da realização de DPN citogenético, este estudo teve por objetivo contribuir para um conhecimento mais preciso acerca da eventual vulnerabilidade psicológica que uma tal instrumentação diagnóstica pode provocar nas grávidas que a ela são submetidas.

Referem-se, em primeiro lugar, como limitações deste estudo, o fato de ter sido conduzido numa amostra relativamente pequena, ademais afetada por uma significativa mortalidade experimental (51.8\%) entre os diferentes momentos de avaliação (embora proporcional entre os grupos), o que poderá condicionar a generalização dos resultados. Esta perda amostral deveuse, sobretudo, ao tipo de estudo (longitudinal com recurso a técnicas audiovisuais) o que tornou mais difícil a adesão das participantes. Outras limitações poderão ser apontadas, nomeadamente a não inclusão de informação relevante acerca do pai e da estrutura do agregado familiar, bem como a não inclusão de outras variáveis valorizadas na literatura (e.g., personalidade, suporte social) no modelo de análise do estudo.

Ainda assim, os resultados do nosso estudo confirmaram a influência que a ansiedade materna e alguns sentimentos relativos à maternidade (irritabilidade, dependência e medos em relação ao bebê) podem exercer sobre a dinâmica interativa mãe-bebê, embora não possa ser excluído que outras variáveis não avaliadas neste estudo (como a capacidade da mulher em lidar com as adversidades psicossociais e biológicas inerentes à gravidez, decorrentes das características da sua personalidade, de fatores biológicos ou de outros acontecimentos) possam, igualmente, influenciar a qualidade da relação mãe-bebê.
Estes resultados permitem fundamentar recomendações no sentido de efetuar uma intervenção profilática que contribua para o desenvolvimento de melhores competências relacionais entre as mães que, durante a gravidez, reagiram com maior ansiedade ao diagnóstico pré-natal.

A gravidez é o momento do ciclo de vida em que o maior número de intervenções preventivas se mostrou efetiva.

Refere-se, a este propósito, que a maioria destas medidas preventivas consiste em identificar um risco determinado e em aplicar o nível de vigilância apropriado com vista à otimização do bem-estar.

Neste contexto, a intervenção de enfermagem tornase necessária e imprescindível na mobilização e desenvolvimento das capacidades da mulher e família para que possam enfrentar e resolver as dificuldades deste período de forma equilibrada.

Ao nível da prevenção assume ainda uma relevância particular a disponibilidade de meios de intervenção psicológica e psicossocial junto destas mulheres, seja pelo seu papel na promoção da adaptação na gravidez e transição para a parentalidade, seja pela sua importância na promoção de uma retaguarda familiar equilibrada para os seus filhos.

A promoção de grupos educativos (preparação para o parto e para a parentalidade e cuidados ao bebê, por exemplo), bem como a visita domiciliária e a implementação de uma linha telefônica de apoio à puérрera, poderão ser medidas de extrema importância para uma prática mais interventiva junto à puérpera na família.

Os dados deste estudo, e as reflexões preventivas e terapêuticas que deles decorrem, justificam que se acentue a realização de investigações semelhantes, a fim de aprofundar a compreensão empírica sobre o modo como as mulheres lidam com as situações de gravidez de risco (em contexto de maternidade tardia) até porque estas situações são cada vez mais frequentes na clínica obstétrica e impõem a cada vez maior refinamento das práticas de enfermagem de saúde materna e obstétrica. 


\section{REFERÊNCIAS}

1. Blickstein I. Motherhood at or beyond the edge of reproductive age. Int J Fertil. 2003;48(1):17-24.

2. INE. Estatísticas Demográficas 2005. Lisboa: Instituto Nacional de Estatística, 2006.

3. Heffner L. Advanced maternal age - How old is too old? N Engl J Med. 2004;351(19):1927-1929.

4. Carolan M, Nelson S. First mothering over 35 years:

questioning the association of maternal age and pregnancy risk. Health Care Women Int. 2007;28(6):534-555.

5. Graça LM. Técnicas invasivas de diagnóstico e terapêutica do feto. In: Graça LM. Medicina Materno Fetal. $4^{\mathrm{a}}$ ed. Lisboa: Lidel; 2010. p.311-315.

6. Öhman SG, Grunewald C, Waldenstrom U. Perception of risk in relation to ultrasound screening for Down's syndrome during pregnancy. Midwifery. 2009;25:264-276.

7. Soubieux MJ, Soulé M. La Psychiatrie Foetale. Paris: PUF; 2005.

8. Kleinveld JH, Timmermans DRM, de Smith DJ, Áder HJ, van der Wal G. Does prenatal screening influence anxiety levels of pregnant women? A longitudinal randomised controlled trial. Prenat Diagn. 2006;26:354-361.

9. Kowalcek I. Stress and anxiety associated with prenatal diagnosis. Best Pract Res Clin Obstet Gynaecol. 2007;21(2):221228.

10. Justo JM. Evolução da ansiedade e dos mecanismos de defesa ao longo da gravidez. [thesis]. Lisboa: Faculdade de Psicologia e de Ciências de Educação/UL; 1994.

11, Catafesta F, Venturi KK, Zagonel IPS, Marialda Martins M. Pesquisa-cuidado de enfermagem na transição ao papel materno entre puérperas. Rev. Eletr. Enf. [Internet]. 2007 [acesso em: 30 jun 2013];9(2):457-75. Disponível em: http://www.fen.ufg.br/revista/v9/n2/v9n2a13.htm.

12. Spielberger CD, Gorsuch I, Lushene RE. Manual for the State Trait Anxiety Inventory. Palo Alto, CA, Consult Psychol Press; 1970.

13. Schaefer ES, Manheimer H. Dimensions of perinatal adjustment. Paper presented at the Eastern Psychological Association Convention; New York; 1960.

14. Schermann L, Hagekull B, Bohlin G, Persson K, Sedin G. Interaction between mother and infant born at risk during the first six months of corrected age. Acta Paediatr. 1997;86:864872.

15. Leithner K, Maar Fischer-Kern M, Hilger E, Loffler-Stastka H, Ponocny-Seliger E. Affective state of women following prenatal diagnosis: predictors of a negative psychological outcome. Ultrasound Obstet Gynecol. 2004;23:240-246.

16. Hewison J, Nixon J, Fountain J, Hawkins K, Jones C, Mason G et al. A randomised trial of two methods of issuing prenatal test results: the ARIA (Amniocentesis Results: Investigation of Anxiety) trial. Int J Obstet Gynaec. 2007;114(4):462-468. 17. Antunes MSC, Patrocínio C. A Malformação do Bebé. Vivências Psicológicas do Casal. Psicologia, Saúde \& Doenças. 2007;8(2):239-252.

18. Grigoletti LVS. A influência da ultrassonografia na representação do filho imaginário - filho real. Psico. Porto Alegre. 2005;36(2):149-157.

19. Brazelton TB, Cramer BG. A relação mais precoce: os pais, os bebés e a interacção precoce. Lisboa: Terramar; 2001.

20. Campos RC. Processo gravídico, parto e prematuridade: uma discussão teórica do ponto de vista do psicólogo. Análise Psicológica. 2000;1(18):15-35.

Artigo recebido em 02/01/2012.

Aprovado para publicação em 04/10/2012.

Artigo publicado em 30/06/2013. 